\title{
Evaluation de la Pollution Métallique des Sédiments Superficiels de la Lagune Potou en Période d'étiage (Littoral de la Côte d'Ivoire)
}

\section{Diangone Eric, Yao Alexis N'guessan, Lou Kala Marie-Claude Toalo, Monde Sylvain, Coulibaly Aoua,}

Laboratoire de Géologie Marine et Sédimentologie, Université de Cocody, Abidjan, Côte d'Ivoire

Submitted: 19 June 2020

Accepted: 27 November 2020

Published: 30 November 2020

Corresponding author:

Diangone Eric

\section{DOI: $10.19044 /$ esj.2020.v16n33p70 \\ (c) Copyright 2020 Diangone et al. \\ Distributed under Creative Common BY-NC-ND 4.0 OPEN ACCES}

Cite as:

Diangone E., Y. N'guessan, L. Toalo, M.Sylvain, CM Aoua (2020). Evaluation de la Pollution Métallique des Sédiments Superficiels de la Lagune Potou en Période d'étiage (Littoral de la Côte d'Ivoire). European Scientific Journal, ESJ, 16 (33), 70. https://doi.org/10.19044/esj.2020.v16n33p70

\begin{abstract}
Resume
La présente étude vise à évaluer la pollution métallique des sédiments superficiels de la lagune Potou (littoral de la Côte d'Ivoire en période d'étiage). La méthode utilisée à cet effet repose en premier sur l'échantillonnage des sédiments et sur la détermination de leurs concentrations en ETM (Élément Trace Métallique) à l'aide de la technique spectrométrique. Ensuite, l'estimation du niveau de la pollution métallique des sédiments, a été faite en comparant les concentrations des métaux obtenues à celles de la croûte continentale de Wedepohl (1995) et à celles des sédiments non pollués de Calamari et Naeve (1994) et Kikouama et al. (2009). De même, l'estimation du niveau de la pollution métallique des sédiments a été réalisée par le calcul de plusieurs indices de pollution dont le facteur d'enrichissement et l'index de géoaccumulation. L'approche méthodologique s'est enfin achevée par l'évaluation des risques des sédiments en place à partir des valeurs guides TEC (Threshold Effects Concentrations) et PEC (Probable Effect Concentration) et par l'étude de la distribution spatiale des différents contaminants au sein de la zone étudiée. Au total, 15 ETM ont été identifiés dans les sédiments du milieu, à savoir l'aluminium (Al), l'arsenic (As), le calcium (Ca), le cadmium (Cd), le chrome (Cr), le cuivre $(\mathrm{Cu})$, le fer $(\mathrm{Fe})$, le potassium $(\mathrm{K})$, le magnésium $(\mathrm{Mg})$, le manganèse $(\mathrm{Mn})$, le sodium $(\mathrm{Na})$, le nickel $(\mathrm{Ni})$, le plomb $(\mathrm{Pb})$, l'antimoine (Sb), et le zinc (Zn). Ces ETM ont des teneurs variables évoluant en moyenne entre 0,81 et $46693,33 \mathrm{mg} / \mathrm{Kg}$. Cette importante variabilité est aussi constatée au niveau des valeurs d'enrichissement en ETM. Celles-ci sont comprises en moyenne entre 0,03 et $4,15 \mathrm{mg} / \mathrm{Kg}$. Concernant l'index de géoaccumulation, les valeurs trouvées sont en
\end{abstract}


European Scientific Journal, ESJ

November 2020 edition Vol.16, No.33

majorité négatives sauf chez le zinc $(0,77 \mathrm{mg} / \mathrm{Kg})$ et l'antimoine $(1,4 \mathrm{mg} / \mathrm{Kg})$. L'ensemble de ces résultats a montré que les sédiments de la lagune Potou sont de mauvaise qualité car pollués par le cuivre, le fer, le zinc et l'antimoine. Ces métaux, en particulier le zinc et l'antimoine, peuvent exercer un effet néfaste sur les organismes aquatiques du milieu lagunaire ainsi que sur l’Homme..

Subject: Geologie

Mots-clés: Pollution, sédiments, lagune Potou, Côte d'Ivoire, ETM, géoaccumulation 


\title{
Evaluation of Metallic Pollution of Surface Sediments in the Potou Lagoon during Low Water (Coast of Côte d'Ivoire)
}

\author{
Diangone Eric, \\ Yao Alexis N'guessan, \\ Lou Kala Marie-Claude Toalo, \\ Monde Sylvain, \\ Coulibaly Aoua, \\ Laboratoire de Géologie Marine et Sédimentologie, Université de Cocody, \\ Abidjan, Côte d'Ivoire
}

DOI: $\underline{10.19044 / \text { esj.2020.v16n33p70 }}$

\begin{abstract}
This paper focuses on evaluating the metallic pollution of the superficial sediments of the Potou lagoon (Coast of Côte d'Ivoire) during the low water period. The method used for this study is based, first of all, on the sampling of the sediments and on the determination of their respective concentrations in MTE (Metal Trace Element) using the spectrometric technique. The estimation of the level of metallic pollution of the sediments was made by comparing the concentrations of the metals obtained with those of the continental Wedepohl crust (1995) and those of the unpolluted sediments of Calamari and Naeve (1994) and Kikouama et al. (2009). It was also achieved by calculating several pollution indices including the enrichment factor and the geo-accumulation index. The methodological approach was finally completed by assessing the risks of sediments in place from the TEC (Threshold Effects Concentrations) and PEC (Probable Effect Concentration) guide values and by studying the spatial distribution of the various contaminants at the site within the study area. A total of 15 MTE were identified in the sediments of the medium, namely: aluminium (Al), arsenic (As), calcium (Ca), cadmium (Cd), chromium (Cr), copper (Cu), iron $(\mathrm{Fe})$, potassium (K), magnesium (Mg), manganese (Mn), sodium (Na), nickel (Ni), lead $(\mathrm{Pb})$, antimony $(\mathrm{Sb})$, and zinc $(\mathrm{Zn})$. These MTE have variable contents ranging on average between 0.81 and $46693.33 \mathrm{mg} / \mathrm{kg}$. This important variability is also found in the MTE enrichment values. These are on average between 0.03 and 4.15. Regarding the geo-accumulation index, the values
\end{abstract}


found are mostly negative except for zinc (0.77) and antimony (1.4). All of these results showed that the sediments of the Potou lagoon are of poor quality because they are polluted by copper, iron, zinc, and antimony. These metals, in particular zinc and antimony, can have a detrimental effect on the aquatic organisms that inhabit the environment as well as humans.

Keywords: Pollution, sédiment, Potou lagoon, Côte d'Ivoire, MTE, geoaccumulation

\section{Introduction}

Dépressions côtières situées au-dessus du niveau moyen des océans (Lankford, 1977), les lagunes représentent environ $13 \%$ de la longueur des côtes du monde. Elles sont en communication plus ou moins étroite et permanente avec la mer et parfois avec un ou plusieurs fleuves. Les lagunes servent par ailleurs de refuge et/ou d'habitats à de nombreuses espèces vivantes grâce à leurs mangroves ; l'un des traits spécifiques qui fait de ces espaces un patrimoine unique qui mérite d'être préservé. Malheureusement, ces milieux naturels censés être protégés sont soumis aujourd'hui à une dégradation accrue. En effet, cette situation trouverait son origine dans le développement croissant à travers le monde des activités socio-économiques dans les zones côtières. Ces attraits s'expliquent par les nombreuses potentialités physiques, biologiques, écologiques et hydrodynamiques de ces zones. En Côte d'Ivoire, la pollution des milieux lagunaires et en particulier de la lagune Potou (Sud-Est du littoral), est devenue un phénomène alarmant qui menace gravement l'environnement car de nombreux éléments chimiques tels que les métaux y sont rejetés incessamment sans précaution. Ces éléments chimiques, dont la gravité de la pollution augmente avec l'emploi des intrants agricoles comme les engrais et les pesticides dans les plantations de la région et particulièrement avec le développement industriel des villes environnantes comme Abidjan ; nécessitent une attention particulière. Cette dernière est non seulement liée à la persistance des métaux dans l'environnement car difficilement éliminés dans l'environnement, ni détoxifiés ou dégradés par les activités métaboliques des organismes vivants qui les ingèrent ; mais aussi due au fait que leurs fortes teneurs sont à l'origine de conséquences néfastes sur les ressources naturelles et l'Homme (Dufour, 1981 ; Marchandet Martin, 1985; Adingra \& Kouassi, 2011; Irié, 2017). La lagune Potou, qui constitue l'une des grandes réserves en eau douce situées à proximité de la ville d'Abidjan, devient donc un danger permanent pour les populations. De nombreuses études ont déjà été initiées sur certaines lagunes du pays en vue de sensibiliser la population et les autorités sur les dangers et l'ampleur de la pollution (travaux de N'Guessan et al., 2009 ; N'Guessan et al., 2013 ; et de Coulibaly et al., 2014; ...). Cependant, ces travaux devraient être approfondis 
en lagune Potou. C'est pourquoi la présente étude vise à évaluer la pollution métallique des sédiments de surface de ladite lagune. Les sédiments peuvent accumuler des substances plus ou moins dégradables, non dégradables, artificielles et/ou naturelles, et devenir de véritables réservoirs de polluants, en particulier pour les éléments traces métalliques (ETM).

\section{Matériel Et Méthodes}

\section{II.1 Présentation de la zone d'étude}

Avec une superficie de l'ordre de $22,8 \mathrm{~km}^{2}$, la zone d'étude est limitée au sud par la sous-préfecture de Bingerville et à l'ouest par la ville d'Abidjan (Figure 1). Elle présente un climat équatorial de transition marqué par quatre principales saisons continentales dont deux grandes et deux petites. Les deux grandes saisons (sèche et pluvieuse) s'étendent de décembre à avril, et de mai à juillet ; tandis que les deux petites saisons (sèche et pluvieuse) se situent d'une part entre août et septembre ; et d'autre part entre octobre et novembre. En ce qui concerne les saisons lagunaires, celles-ci au nombre de trois évoluent respectivement de janvier à avril (saison sèche), de mai à aout (saison des pluies) et enfin de septembre à décembre (saison des crues). Les sols du secteur d'étude sont de type ferralitique lessivé (sols à sesquioxydes) et des sols hydromorphes. La végétation quant à elle est constituée de la forêt dense sempervirente ou ombrophile à laquelle s'ajoute un autre type de forêt marécageuse, avec des bambous et des mangroves en bordures de lagune. Concernant les apports en eau douce de la région étudiée, ils sont assurés principalement par la Comoé, la Mé, et l’Agnéby. La Comoé, qui débouche à l'extrémité orientale du système Ebrié, fournit l'essentiel (80 \% du volume total), avec un régime quasi tropical de transition. L'apport annuel de la Mé est de l'ordre de six fois le volume du système Potou-Adjin dans lequel elle se déverse. La Mé s'apparente avec l’Agnéby au régime équatorial de transition. Ces apports sont complétés par ceux des petites rivières d'importance très médiocre, disséminées sur la rive Nord, et par ceux des précipitations et bien entendu les apports négatifs de l'évaporation. 


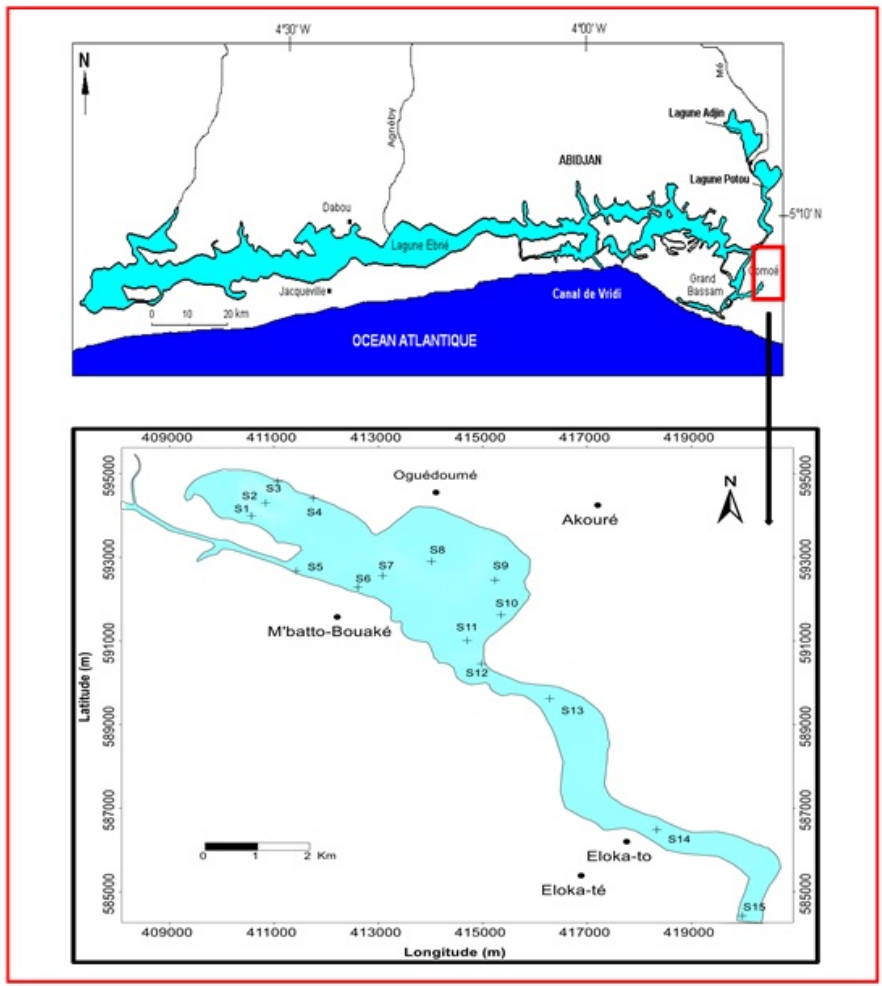

Figure 1. Présentation de la zone d'étude et des stations de prélèvements des sédiments

\section{II.2 Méthodologie de l'étude}

\section{II.2.1 Echantillonnage des sédiments superficiels}

L'échantillonnage des sédiments a été réalisé sur l'ensemble de la lagune Potou, les 13 et 15 au 20 avril 2017, au moyen d'une benne Van Veen. Le nombre total de sédiments prélevés s'élève à 15 (Figure 1).

\section{II.2.2 Analyses chimiques des sédiments}

\section{II.2.2.1 Dosage des ETM par spectrométrie d'absorption atomique à flamme}

Le dosage des ETM a débuté par une préparation physique, durant laquelle les sédiments échantillonnés ont été séchés (à l'étuve) pendant 24 heures et à $40^{\circ} \mathrm{C}$; puis broyés, homogénéisés, et enfin tamisés sur des toiles de maille de $2 \mathrm{~mm}$ et $63 \mu \mathrm{m}$. Cette phase préalable a laissé place ensuite au dosage des différents ETM présents dans les sédiments, à l'aide de la méthode spectrométrique d'absorption atomique à flamme. Cette technique de 
spectrométrie utilisée repose essentiellement sur le couplage d'une torche à plasma ICP (Inductively Coupled Plasma) générant des ions monovalents positifs et d'un spectromètre de masse quadripolaire qui sépare ces ions en masse.

\section{II.2.2.2 Estimation de l'intensité de pollution}

Les valeurs références choisies dans cette étude pour estimer le niveau de contamination des sédiments sont celles de la croûte continentale (Wedepohl, 1995) et celles des sédiments non pollués (Calamari \& Naeve, 1994 ; Kikouama et al., 2009) (Tableau I).

Tableau I. Concentrations des ETM dans la croûte continentale (Wedepohl, 1995) et dans les sédiments non pollués (Calamari \& Naeve, 1994 ; Kikouama et al., 2009)

\begin{tabular}{|c|c|c|c|c|c|c|c|c|c|c|c|c|c|c|c|}
\hline ETM (mglkg) & Al & As & $\mathrm{Ca}$ & Cd & $\mathrm{Cr}$ & $\mathrm{Cu}$ & $\mathrm{Fe}$ & $\mathrm{k}$ & $\mathrm{Mg}$ & $\mathrm{Mn}$ & $\mathrm{Na}$ & $\mathrm{Ni}$ & $\mathrm{Pb}$ & sb & $2 n$ \\
\hline $\begin{array}{l}\text { UCC (Upper Continental } \\
\text { Crust) }\end{array}$ & 77440 & 2 & 28450 & 0.1 & 35 & 14 & 30880 & 28650 & 13510 & 527 & 25670 & 18 & 17 & 0,31 & 52 \\
\hline \multicolumn{16}{|l|}{ Calamari et Naeve, (1994) } \\
\hline & - & - & - & 0,11 & - & 33 & 41000 & . & . & . & . & . & 18 & $\cdot$ & 85 \\
\hline Kikouama et al., (2009) & & & & & & & & & & & & & & & \\
\hline & - & - & . & 0,44 & . & 24,84 & - & . & - & - & - & . & 12,88 & - & 45,13 \\
\hline
\end{tabular}

L'impact des métaux polluants sur la qualité des sédiments a été aussi chiffré à travers le calcul des indices de pollution :

- Le facteur d'enrichissement (FE) est établi par la relation de Sutherland (2000): $\mathbf{F E}=([\mathrm{M}]$ éch/ [M']éch )/(M]RM/ [M']RM); Avec : FE = facteur d'enrichissement; [M]éch = concentration du métal $M$ dans l'échantillon; [M']éch = concentration de l'élément normalisateur dans l'échantillon; $[\mathrm{M}] \mathrm{RM}=$ concentration du métal $\mathrm{M}$ dans le matériau de référence et [M']RM = concentration de l' élément normalisateur dans le matériau de référence. Le fer $(\mathrm{Fe})$ a été choisi comme élément normalisateur en raison de son abondance naturelle dans l'environnement.

- L’index de géo-accumulation est donné par la relation Müller (1969): Igeo = Log2 $(\mathrm{Cn} / 1,5 \mathrm{Bn})$; Avec : Igeo = indice de géo-accumulation; log2 $=$ logarithme de base $2 ; \mathrm{n}=$ élément considéré $; \mathrm{Cn}=$ concentration mesurée dans l'échantillon ; $\mathrm{B}=$ fond géochimique ; 1,5 = constante qui associe les variations naturelles de la concentration d'un élément dans un milieu et les faibles pressions anthropiques.

\section{II.2.2.3 Évaluation de la qualité environnementale des sédiments}

La qualité environnementale des sédiments a été évaluée à partir des valeurs TEC et PEC de Macdonald et al. (2000). La TEC (Threshold Effects Concentration) désigne les limites de concentrations des contaminants en dessous desquelles les organismes vivants ne sont pas menacés. La PEC 
(Probable Effect Concentration) représente les concentrations des contaminants au-dessus desquelles ceux-ci ont un effet sur les organismes vivants (Tableau II).

Tableau II. Valeurs de TEC et de PEC (Macdonald et al., 2000)

\begin{tabular}{|l|l|l|l|l|l|l|}
\hline ETM & $\mathrm{Cu}$ & $\mathrm{Cr}$ & $\mathrm{Zn}$ & As & $\mathrm{Cd}$ & $\mathrm{Pb}$ \\
\hline $\begin{array}{l}\text { TEC } \\
(\mathrm{mg} / \mathrm{kg})\end{array}$ & 31 & 43,3 & 121 & 9,79 & 0,99 & 35,6 \\
\hline PEC(mg/kg) & 149 & 111 & 459 & 33 & 4,98 & 128 \\
\hline
\end{tabular}

\section{II.2.2.4. Étude de la répartition spatiale des polluants métalliques}

Elle a été faite à partir du logiciel Surfer 11 à partir du fichier de données Excel comportant les coordonnées GPS (longitudes et latitudes) de chaque station de prélèvement. Ces données permettent de réaliser des fichiers mathématiques en utilisant l’interpolation krigeage linéaire.

\section{Résultats Et Interprétations}

\section{III.1 Teneurs en ETM des sédiments}

Les concentrations des ETM obtenues après dosage, dans les sédiments de chacun des 15 sites de prélèvement, évoluent en moyenne entre $0,81 \mathrm{mg} / \mathrm{kg}$ et $46693,33 \mathrm{mg} / \mathrm{kg}$. Ces résultats mettent en exergue une variabilité importante des teneurs métalliques.

\section{III.2 Estimation de l'intensité de pollution métallique}

$\square$ En fonction des teneurs des éléments traces métalliques

Les résultats observés (Tableau III) montrent que les ETM tels que le Ni, $\mathrm{Al}, \mathrm{K}, \mathrm{Na}, \mathrm{Ca}, \mathrm{Mg}, \mathrm{Pb}$ et $\mathrm{Cd}$, ont des concentrations inférieures aux valeurs de la croûte continentale dans les sédiments de la zone d'étude. Ces faibles valeurs témoignent d'une dilution qui s'opère par des apports de sédiments moins contaminés ou non. D’autres métaux comme $\mathrm{Cu}$, As, $\mathrm{Cr}, \mathrm{Mn}, \mathrm{Zn}, \mathrm{Sb}$ et Fe présentent des teneurs plus élevées, ce qui traduit par conséquent une contamination polymétallique des sédiments superficiels par ces métaux. Cette contamination pourrait s'expliquer par les rejets dans le milieu lagunaire, des déchets non traités, émanant des diverses activités domestiques, agricoles et industrielles réalisées tout autour de la lagune. Les ETM dont le $\mathrm{Cu}, \mathrm{Fe}, \mathrm{Zn}$ appartenant à ce dernier groupe, montrent en plus des concentrations supérieures à celles des sédiments non pollués. Ceci au niveau des échantillons des stations prélèvement 15 pour le cuivre ; 2, 5, 8, 10,12, 14 et 15 pour le fer et pour le zinc, les échantillons 1, 2, 5, 8, 10, 11, 12, 13 ,14 et 
15 (Figure 2). Cette situation met en évidence une pollution des sédiments de la zone étudiée par ces trois éléments métalliques.

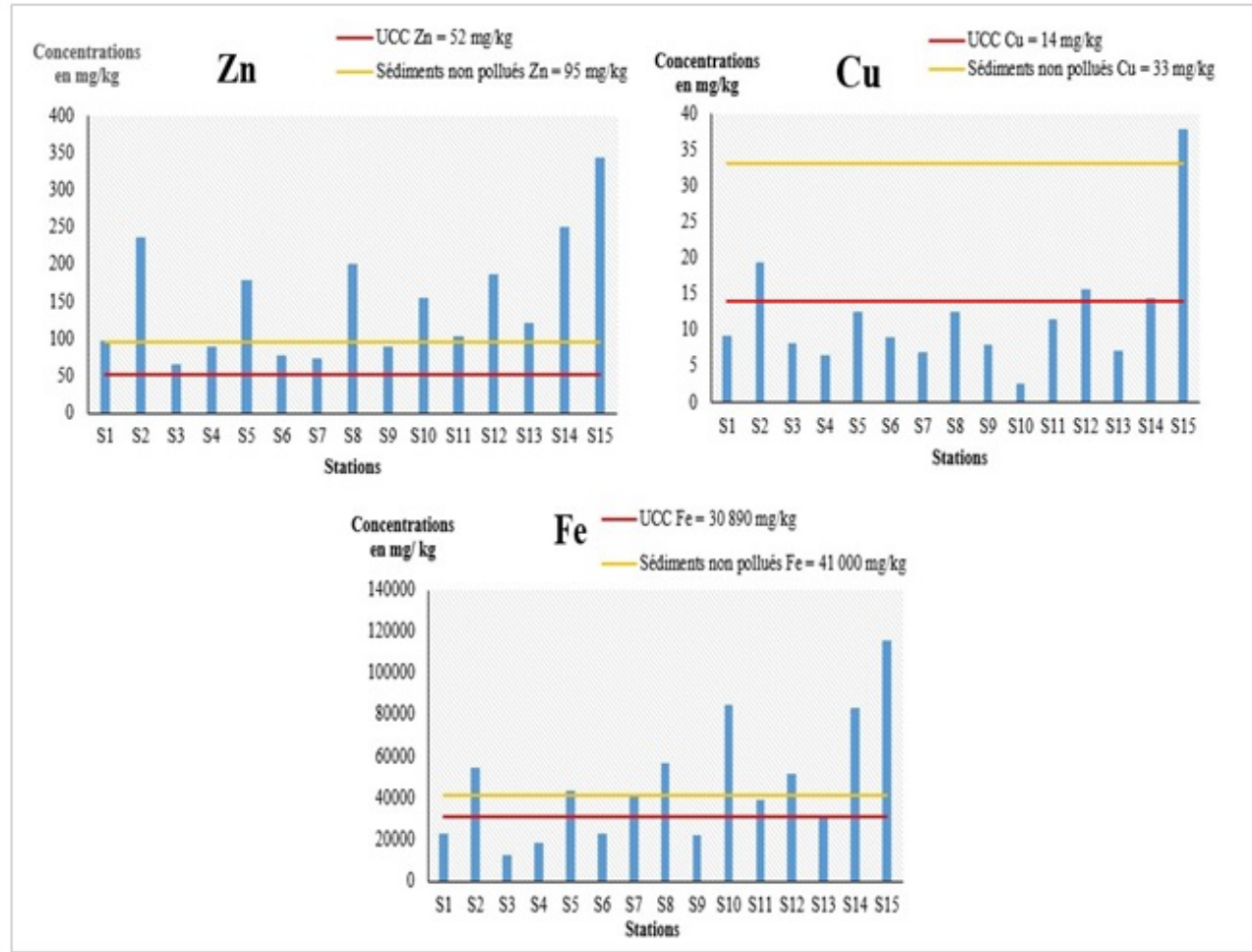

Figure 2. Evolutions des teneurs en Zn, Cu et Fe dans les sédiments étudiés

$\square$ En fonction des indices de pollution

L'évolution de l'enrichissement en aluminium dans les sédiments se fait entre 0,001 et 0,05 avec un taux moyen de 0,03. Les valeurs d'enrichissement en arsenic évoluent de 0,04 à 0,49 , soit une grandeur moyenne de 0,22 . Elles sont inférieures à 1 dans l'ensemble des sédiments étudiés. Au niveau du calcium, des valeurs situées entre 0,01 et 0,23 sont observées. La moyenne d'enrichissement en Calcium s'élève par contre à 0,07 . L'enrichissement en calcium reste également inférieur à 1 dans les sédiments. L'enrichissement en cadmium se fait entre 0,13 et 1,21 avec une moyenne de 0,47 . Seul l'échantillon 3 présente un enrichissement supérieur à 1. Les sédiments de la zone d'étude ont un enrichissement moyen en chrome de 0,44 . La valeur d'enrichissement la plus élevée est égale à 0,77 . La valeur la plus faible atteint 0,09. L'enrichissement en chrome n'excède pas 1 dans tous les sédiments étudiés. Le cuivre a un enrichissement maximal équivalent à 1,41 et un minimal égal à 0,07 . La moyenne d'enrichissement est équivalente à 0,67 . Les valeurs supérieures à 1 sont observées seulement au niveau de l'échantillon 3. L'enrichissement en potassium oscille entre 0,005 
et 0,11 , soit une moyenne de 0,06 . La variation de l'enrichissement en magnésium se fait entre 0,06 et 0,40 avec une moyenne de 0,18 . Cette variation est inférieure à $1(\mathrm{FE}<1)$ dans l'ensemble des échantillons. Pour ce qui est du manganèse, les résultats révèlent des valeurs comprises entre 0,15 et 0,60 , d'où une moyenne de 0,33 . L'enrichissement en manganèse demeure inférieur à 1 dans la totalité des sédiments étudiés. Ceux-ci présentent un enrichissement en sodium inférieure à 1 . Cet enrichissement évolue de 0 à 0,10 , avec une moyenne de 0,05 . Le facteur d'enrichissement en nickel évolue entre 0,07 et 0,57 avec une moyenne de 0,28 (FE $<1)$. L'enrichissement en plomb des sédiments de la lagune Potou varie de 0,001 à 0,31, avec une moyenne de 0,13 . Cet enrichissement est inférieur à 1 dans l'ensemble des échantillons étudiés. L'antimoine présente un enrichissement oscillant entre 0,22 et 7,85 avec une moyenne de 4,15. Ce enrichissement reste inférieur à 1 dans les sédiments 3 et 5 ; compris entre 1 et 3 dans les échantillons 4 et 6 ; entre 3 et 5 dans les sédiments 7,8,9,10,11 et 15 et un enrichissement supérieur à 5 au niveau des échantillons 5,2,12,13 et 14 .Quant au zinc, la variation de son enrichissement se fait entre 1,07 et 3,08, avec une moyenne de 2,12. Seuls les sédiments 7, 10, 11,14 et 15 ont un enrichissement en zinc inférieur à 2 (Figure 3).

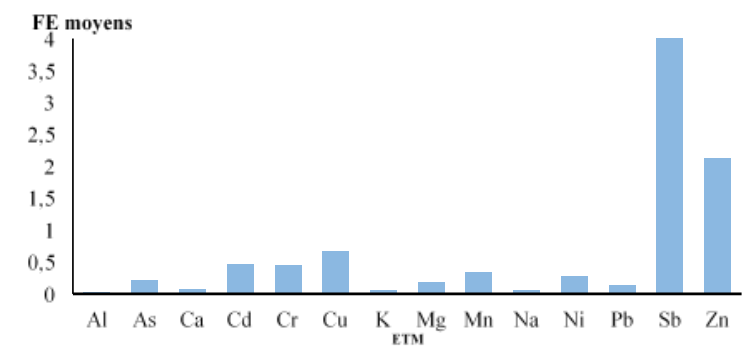

Figure 3. Variation des valeurs moyennes du facteur d'enrichissement

Ces résultats suggèrent deux origines possibles des ETM, à savoir l'origine naturelle terrigène et l'origine anthropique. La première origine regroupe les métaux à facteur d'enrichissement inférieur à 1 . Ce sont le $\mathrm{Pb}$, As, $\mathrm{Cr}, \mathrm{Ni}, \mathrm{Al}, \mathrm{Mn}, \mathrm{K}, \mathrm{Mg}, \mathrm{Ca}, \mathrm{Na}, \mathrm{Cd}$ et le $\mathrm{Cu}$. Un tel facteur indique une absence d'enrichissement ou un enrichissement minimal en ETM. La seconde origine est caractérisée par des facteurs d'enrichissement supérieurs à 1 (FE > 1 ), c'est le cas du Zn $(2,12)$ et $\mathrm{Sb}(4,15)$ (Figure 3). Ces forts enrichissements, probablement issus du transport par les cours d'eau des déchets agricoles et industriels, mettent en relief un enrichissement modéré en zinc et antimoine des sédiments lagunaires. Toutefois, l'hypothèse d'un enrichissement significatif en Sb n'est pas épargnée avec son maximum d'enrichissement égale à $7,85(5<\mathrm{FE}<10)$. 


\section{$\square$ Indice de géo accumulation}

Les indices de géo accumulation des ETM dans les sédiments de la lagune Potou évoluent pour l'aluminium de -7,09 à - 4,27 avec une moyenne de $-5,45$. Ces indices varient respectivement pour l'arsenic entre - 4,32 et 0,30 , soit une moyenne de $-2,62$. Pour ce qui est du calcium, l'Igéo oscille de $-6,51$ à -2,95, avec un taux moyen de - 4,97. L'Igéo du cadmium est constant à sa valeur de $-1,58$. Le chrome a un Indice de géo accumulation variant entre $-2,71$ et - 0,29 et en moyenne égal à -1,56. Les ETM tels que le cuivre, le fer, le potassium et le magnésium ont des variations comprises respectivement entre $-2,88$ et 1,$01 ;-1,86$ et 1,$32 ;-6,80$ et $-2,92$; entre - 4,16 et $-1,33$ avec des moyennes de $-0,88,-0,24,-4,67$ et de $-2,70$. L'Igéo du manganèse fluctue entre $-4,03$ et $-0,25$, avec une moyenne de $-1,96$. L'Igéo minimal du sodium est -15,26 et le maximum est de -3,09. La moyenne est équivalente à -5,40. D'autres ETM comme le nickel présentent un indice évoluant entre -3,25 et 0,92 avec une moyenne de $-2,21$. Celui du plomb varie de $-8,99$ à $-1,43$ et en moyenne de $-3,90$. L'antimoine a un Igéo allant de $-3,22$ à 3,39 avec une moyenne de 1,40. Enfin, les valeurs de l'Igéo du zinc varient entre -0,23 et 2,14 , soit une valeur moyenne égale à 0,77 . Les valeurs d'Igéo négatives (Igéo < 0) de certains métaux comme $\mathrm{Pb}, \mathrm{Cr}, \mathrm{Ni}, \mathrm{Al}, \mathrm{K}, \mathrm{Mn}, \mathrm{Mg}, \mathrm{Na}, \mathrm{Cd}, \mathrm{Ca}$, $\mathrm{As}, \mathrm{Cu}, \mathrm{Fe}$ impliquent que les sédiments étudiés ne sont pollués par ces métaux. L'Igéo moyen du zinc compris entre 0 et $1(0 \leq$ Igéo $<1)$ traduit une absence de pollution à une pollution modérée des sédiments par ce métal. L'Igéo de l'antimoine $(1,4)$ oscillant entre 1 et $2(1 \leq$ Igéo $<2)$ montre une pollution modérée des sédiments.

\section{III.3 Qualité environnementale des sédiments}

Les résultats relatifs à la qualité environnementale des sédiments indiquent en ce qui concerne les valeurs guides TEC que $100 \%$ des teneurs du plomb, de l'arsenic, du cadmium, du nickel, du chrome sont inférieures à la valeur référence. Il en de même que 93 ,33\% des teneurs du cuivre. Le zinc par contre présente 46,67\% de ses concentrations en dessous du TEC référence. Concernant le PEC, les concentrations du plomb, cuivre, arsenic, cadmium, nickel, chrome et le zinc sont toutes inférieures au PEC standard. Ces résultats révèlent $0 \%$ des teneurs du plomb, du cuivre, de l'arsenic, du cadmium, du nickel, du chrome et 53,33 \% des teneurs du zinc comprises entre le TEC et le PEC. Pour le reste des métaux de la zone d'étude, la qualité environnementale n'a pu être malheureusement évaluée en raison de leurs valeurs guides TEC et PEC encore indéterminées (Tableau III et Figure 4). 
European Scientific Journal, ESJ

November 2020 edition Vol.16, No.33
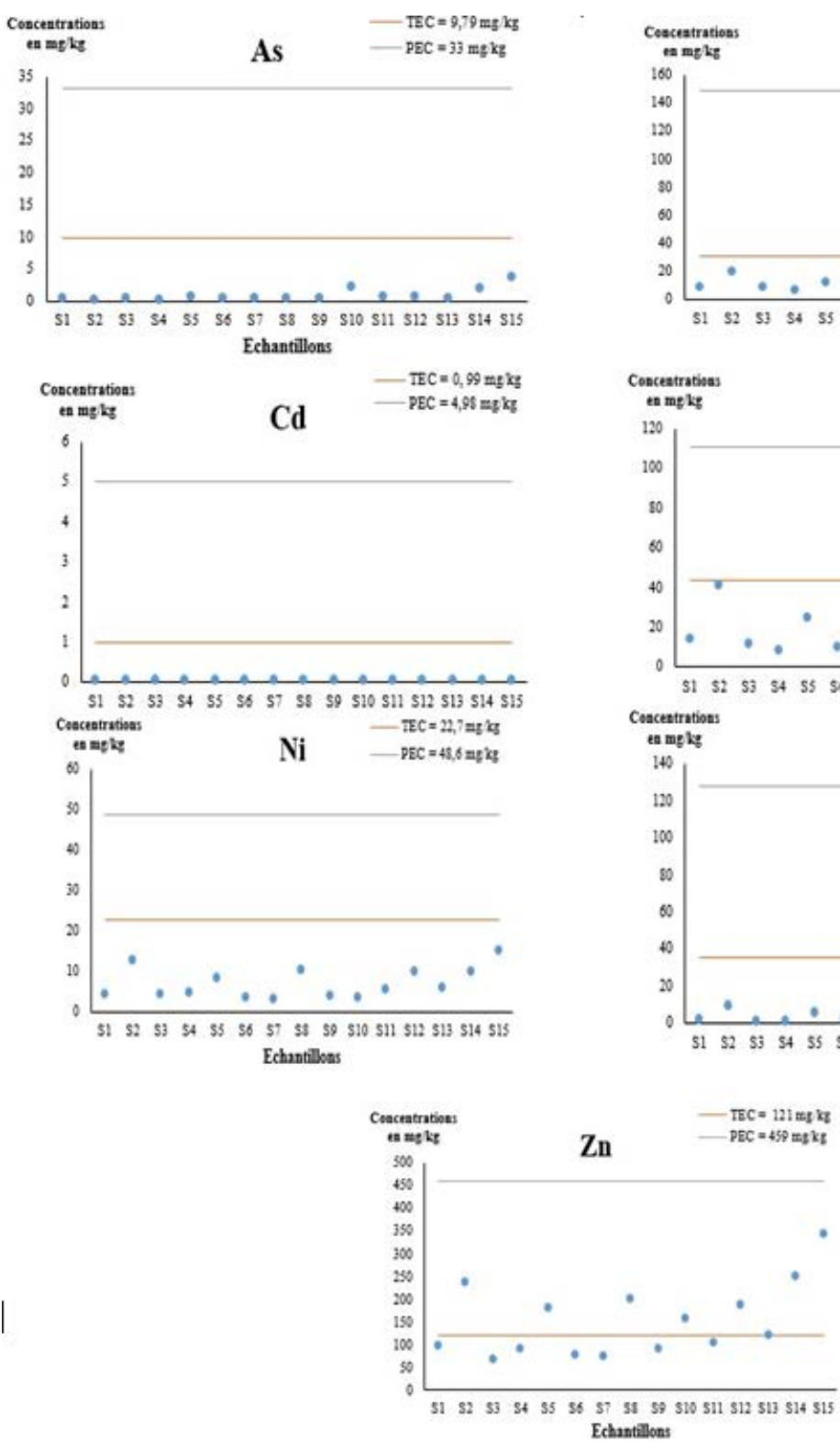

Figure 4. Teneurs en ETM des sédiments comparées aux valeurs guides d'effets biologiques (TEC et PEC)

Tableau III. Proportion (mg/kg) des sédiments par rapport au TEC et PEC

\begin{tabular}{|c|c|c|c|c|c|c|}
\hline & $\mathrm{Cd}$ & $\mathrm{Pb}$ & $\mathrm{As}$ & $\mathrm{Cr}$ & $\mathrm{Cu}$ & $\mathrm{Zn}$ \\
\hline TEC & 0,99 & 35,6 & 9,79 & 43,3 & 31 & 121 \\
\hline PEC & 4,98 & 128 & 33 & 111 & 149 & 459 \\
\hline \% inférieur au TEC & 100 & 100 & 100 & 100 & 93,33 & 46,67 \\
\hline
\end{tabular}




\begin{tabular}{|c|c|c|c|c|c|c|}
\hline \% entre TEC et PEC & 0 & 0 & 0 & 0 & 0 & 53,33 \\
\hline \% supérieur au PEC & 0 & 0 & 0 & 0 & 0 & 0 \\
\hline
\end{tabular}

\section{III.4 Répartition spatiale des ETM polluants}

La distribution du zinc sur l'ensemble de la lagune Potou est relativement hétérogène (écart-type de 80,76). Ce métal a des concentrations n'excédant pas $500 \mathrm{mg} / \mathrm{kg}$. Les fortes valeurs allant de 200 à $344 \mathrm{mg} / \mathrm{kg}$ rencontrées de ce métal s'observent notamment dans la partie sud du plan d'eau, plus précisément au niveau du bras lagunaire menant à la lagune Ebrié. Elles se situent également au voisinage de la localité d'Oguédoumé et dans une infime portion au nord-ouest (chenal menant à la lagune Adjin). Les plus faibles et moyennes teneurs oscillantes entre 66 et $200 \mathrm{mg} / \mathrm{kg}$ sont remarquées le long des berges au nord et dans la partie centrale proche de M'BattoBouaké. Le fer est le métal le plus concentré dans les sédiments étudiés. Il se reparti également de façon hétérogène. Son écart-type est de 29036,96, au sein de la zone d'étude. Cet ETM est d'une part fortement concentré au centre-est et à l'extrémité sud-est du bras lagunaire; et d'autres part faiblement concentré au nord et le long du plan lagunaire de M'Batto- Bouaké à l'avant Eloka-to. Les concentrations du métal cuivre sont faibles tout au long de la lagune à l'exception de l'entrée du bras lagunaire en relation avec la lagune Ebrié. Dans cette partie du domaine lagunaire s'observent les valeurs maximales qui atteignent $37 \mathrm{mg} / \mathrm{kg}$. La répartition du cuivre est donc hétérogène avec un écart-type de 8,29.

La répartition latérale de l'antimoine dans les sédiments de la lagune Potou suit la même dynamique que celle du fer avec un écart-type équivalent à 1,5. Les valeurs maximales oscillent entre 3 et 4,89 et les valeurs minimales sont comprises entre 0 et $3 \mathrm{mg} / \mathrm{kg}$ (Figure 5). 
Figure 5. Cartes de répartition spatiale des ETM (Cu, Sb, Fe et Zn)
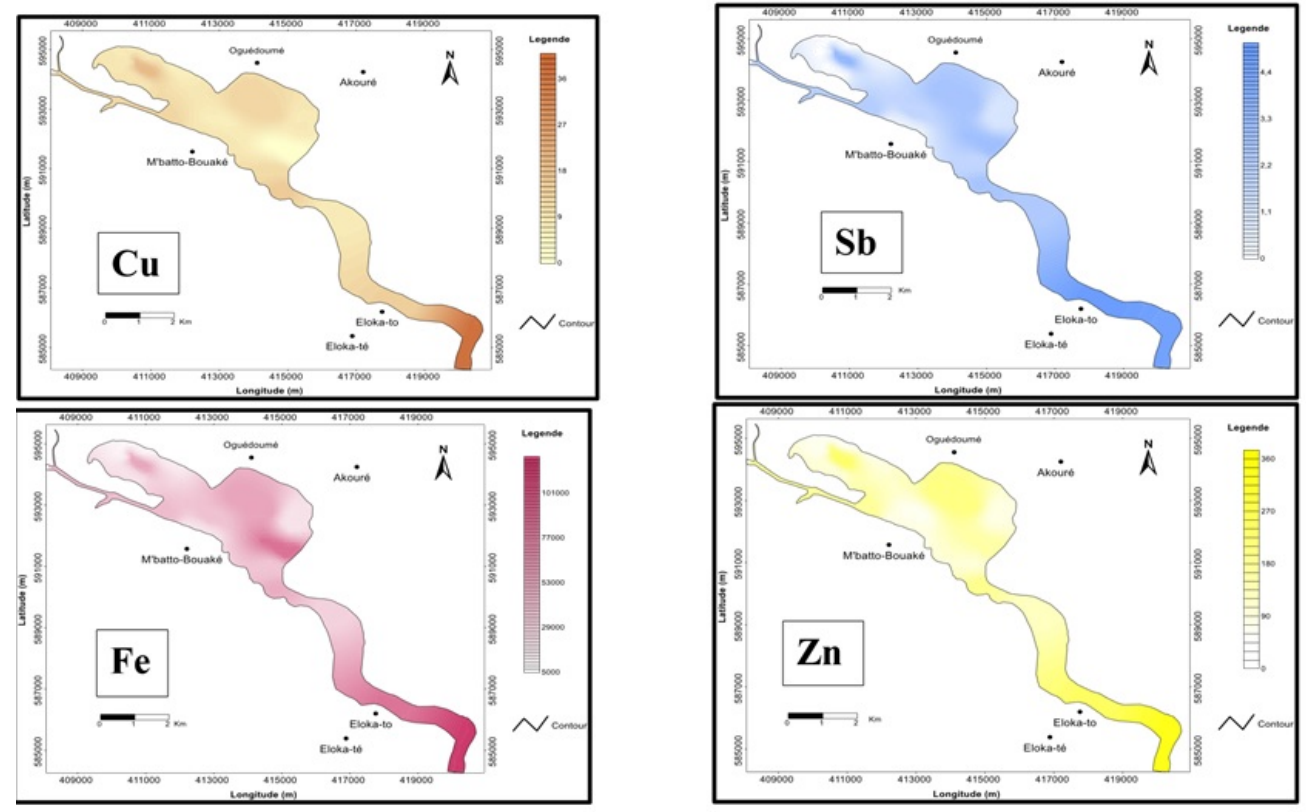

\section{Discussion}

L'évaluation des teneurs moyennes des métaux de cette étude, par comparaison avec celles de la croûte continentale supérieure (UCC) de Wedepohl (1995), montre une contamination polymétallique des sédiments de la lagune Potou par $\mathrm{Cu}, \mathrm{As}, \mathrm{Cr}, \mathrm{Mn}, \mathrm{Zn}, \mathrm{Sb}$ et Fe. Ces valeurs anormales pourraient être dues, selon Keumean et al. (2013), à la charge polluante provenant des différents rejets issus des activités agricoles, domestiques et artisanales. À ces apports s'ajoute la charge contaminante des nombreux effluents d'extractions minières et industrielles. Parmi ces contaminants, le cuivre, le fer et le zinc, qui ont des concentrations supérieures à celles recommandées pour les sédiments non pollués par Calamari et Naeve (1994), contribuent effectivement à la pollution des sédiments lagunaires. Ces métaux constituent par conséquent un danger pour l'eau, les espèces vivantes (végétales et animales) et pour la santé de l'Homme. Les teneurs en métaux ont été comparées à celles étudiées par Chouti et al. (2011) dans les sédiments de la lagune de Porto-Novo, au sud du Bénin. Les concentrations de fer et de manganèse dans les sédiments de la lagune Potou sont les plus élevées. Elles évoluent environ entre 328 et $555 \mathrm{mg} / \mathrm{kg}$ pour Fe et entre 80 et $118 \mathrm{mg} / \mathrm{kg}$ pour Mn. Par contre, au niveau des ETM du Cr, Cd et $\mathrm{Pb}$, les concentrations de la lagune Potou sont moins élevées que celles de la lagune de Porto-Novo (Cr : $91 \sim 210 \mathrm{mg} / \mathrm{kg}$; Cd : 5,39 7,33 mg/kg ; Pb : 3,52 60,62 mg/kg). Les teneurs en chrome, manganèse, zinc et cuivre sont différentes de celles obtenues par Soro et al. (2009) dans les sédiments de la lagune Ebrié (Cr : 8,2 
1618, 6 mg/kg ; Mn: 42,3 415,0 mg/kg ; Zn: 21,6 1081,7 mg/kg ; Cu : 4,8 $\sim 217,4 \mathrm{mg} / \mathrm{kg}$ ). L'évolution des différentes concentrations métalliques met en évidence la présence de deux zones de contamination au sein de la zone d'étude. La première zone en amont (nord et centre) est marquée par les influences de la lagune Adjin et de la Mé. La seconde zone en aval (extrémité sud) est dominée essentiellement par les apports de la lagune Ebrié et du fleuve Comoé. La lagune Ebrié qui partage le même système lagunaire que la lagune Potou contribue de façon significative à la pollution observée. L'étude de l'enrichissement en ETM révèle que la plupart des métaux étudiés ont une variation allant d'un enrichissement nul à un enrichissement faible $(\mathrm{FE}<2)$ excepté le zinc et l'antimoine qui traduisent un enrichissement modéré (FE entre 2 et 5). Ces concentrations sont également confirmées par leurs valeurs d'Igéo respectives de 0,77 et de 1,40 suggérant une absence de pollution à une pollution modérée. L'indice de géo accumulation négatif des autres ETM étudiés indique, selon Müller (1969), que ceux-ci ne constituent aucune menace de pollution pour les sédiments. Pour le nickel (Ni), le FE de la lagune Potou est moins élevé que celui du chenal est de la lagune Ebrié, selon Irié (2017) (FE (Ni) : 0,53 7,06). Il en est de même pour les FE de l'arsenic et du chrome au niveau de la rade portuaire d'Abidjan (Coulibaly et al., 2014). Concernant l'indice de géo accumulation, les métaux tels que le zinc, le cadmium, le cuivre et le plomb sont considérés en lagune Ebrié, comme les polluants les plus importants des sédiments (Irié, 2017). Ces divergences pourraient s'expliquer par le fait que la lagune Potou est une lagune rurale, donc moins affectée par les activités industrielles ; contrairement à la lagune Ebrié qui se situe en milieu urbain. Il ressort de l'évaluation de la qualité des sédiments que la lagune Potou est essentiellement menacée par le zinc. Ce métal présente moins de $75 \%$ de ces teneurs en dessous du TEC, et 53,33\% comprises entre le TEC et le PEC, contrairement aux autres métaux qui ont plus de $75 \%$ des teneurs inferieures au TEC, moins de $75 \%$ supérieures au PEC standard et $0 \%$ comprises entre le TEC et le PEC. Cet ETM constitue donc un danger pour le milieu et en particulier pour les organismes benthiques. Selon Environnement Canada (1997), les effets biologiques engendrés comprennent une diminution de l'abondance des invertébrés benthiques et de la fécondation, un accroissement de la mortalité, la létalité, des modifications comportementales et un développement anormal dans les premiers stades de la vie des organismes benthiques. Par ailleurs, chez l'Homme cela pourrait aussi entraîner des atteintes au système nerveux, des effets myocardiques, des cancers et bien d'autres inconvénients (Charbonnier, 2014 ; INERIS, 2012). Cependant, le zinc n'est pas le seul métal en état de nuire à la qualité environnementale des sédiments. Des études menées dans les sédiments de la rade portuaire d'Abidjan par Coulibaly et al. (2014) et par Irié (2017) au niveau du chenal est de la lagune Ebrié, ont mis en évidence l'impact négatif 
d'autres métaux tels que $\mathrm{Cr}, \mathrm{Ni}, \mathrm{Cd}, \mathrm{Pb}$, As et $\mathrm{Cu}$ en zone urbaine d'Abidjan, fortement polluée par les rejets industriels (Metongo et al., 1993).

\section{Conclusion:}

Les milieux aquatiques sont l'objet de multiples atteintes provoquées par les activités humaines, développées essentiellement le long des côtes. Ces activités génèrent des quantités remarquables de micropolluants, notamment des micropolluants métalliques. Ces polluants sont particulièrement redoutés en raison de leur persistance dans l'environnement et leurs conséquences sur la santé des êtres vivants. En Côte d'Ivoire, l'état alarmant de la situation, en particulier dans les milieux lagunaires du pays, a suscité la réalisation de cette étude. Son objectif principal était d'évaluer la pollution métallique des sédiments superficiels de la lagune Potou, située dans le Sud-Est du pays et en relation avec la lagune Ebrié sujette à la pollution urbaine et industrielle. L'approche méthodologique utilisée à cet effet a permis de mettre en évidence au terme de l'étude, une pollution des sédiments de la zone étudiée essentiellement par le cuivre, le fer, l'antimoine et le zinc. Ces métaux sont issus des rejets des activités domestiques, agricoles et industrielles existants autour de la lagune. Cette situation peut exercer un effet néfaste sur les organismes benthiques mais aussi chez l'Homme.

\section{Liste Des Abréviations}

AFNOR : Association Française de Normalisation

CRE : Centre de Recherche en Écologie

ETM : Élément Trace Métallique

FIT : Front Intertropical

GPS : Global Positioning System

LMD : Maîtrise Doctorat

PHE : Potentially Harmful Elements

STRM : Science de la Terre et des Ressources Minières

UFHB : Université Felix Houphouët -Boigny

UFR : Unité de Formation et de Recherche

OMS : Organisation Mondiale de la Santé

\section{References:}

1. Calamari, D. \& Naeve, H. (1994). Revue de la pollution dans l'environnement aquatique africain. Document Technique du CPCA, No25. Rome, FAO, 129 p.

2. Charbonnier, E. (2014). Caractérisation et interprétation des teneurs naturelles en éléments traces métalliques et métalloïdes sur le territoire 
du SAN Ouest Provence, Mémoire de Stage, Université de Bourgogne, $46 \mathrm{p}$.

3. Coulibaly, A. S., Touré, M., Diangoné, E., Monde, S., Kouamé, A. \& Blanc, G. (2014). Impacts des rejets accidentels sur la qualité environnementale des sédiments de la rade portuaire d'Abidjan (lagune Ebrié ; Côte d’Ivoire). Int. J. Biol. Chem. Sci., Vol. 8, № 6, pp. 2842-2848.

4. Chouti, W., Mama, D., Alassane, A., Changotade, O., Alapini, F., Boukari, M., Aminou, T. \& Afouda, A. (2011). Caractérisation physicochimique de la lagune de Porto Novo (sud Bénin) et mise en relief de la pollution par le mercure, le cuivre et le zinc. Journal of Applied Biosciences, vol. 43, pp 2882 - 2890.

5. Durand, J.R. \& Skubich, M. (1982). Les lagunes ivoiriennes. Aquaculture, 27, pp. 211- 250.

6. Environment Canada (1997). Canadian sediment quality guidelines for cadmium: Supporting document. Service de la conservation de l'environnement, Direction générale de la science des écosystèmes, Direction de la qualité de l'environnement et de la politique scientifique, Division des recommandations et des normes, Ottawa.

7. Guiral, D. (1992). L'instabilité physique, facteur d'organisation et de structuration d'un écosystème tropical saumâtre peu profond : la lagune Ebrié. Milieu de Vie, vol. 42, №2, pp 73-92.

8. INERIS (2012). Manganèse et dérivés. Fiche de données toxicologiques et environnementales des substances chimiques, $81 \mathrm{p}$.

9. Irie, B. T. J-G. (2017). Caractérisation de l'interface eau-sédiment dans un environnement lagunaire à forçage : hydrologie et évaluation environnementale du chenal est dela lagune

10. Ebrié (côte d'ivoire). Thèse Doctorat, Université Félix Houphouët Boigny (Côte d'Ivoire), 215p.

11. Keumean, K.N., Bamba, S.B., Soro, G., Soro, N., Metongo, B.S. \& Biemi, J. (2013). Concentration en métaux lourds des sédiments de l'estuaire du fleuve Comoé à GrandBassam (Sud-Est de la Côte d’Ivoire). J. Appl. Biosci., Vol. 61, pp 4530 - 4539.

12. Kikouama, O.J.R., Konan, K.L., Katty, A., Bonnet, J.P., Balde, L. \& Yagoubi, N. (2009). Physicochemical characterization of edible clays and release of trace elements. Appl. Clay Sci., Vol.43, Nº1, pp. 135141. 
13. Macdonald, D.D., Ingersoll, C.G. \& Berge, T.A. (2000). Development Freshwater and Ecosystems Evaluation of Consensus-Based Sediment Quality Guidelines for freshwater and Ecosystems. Arch. Env. Cont. Toxicol., 39, pp20-31.

14. Metongo, B.S., Kouassi, A.M. \& Kaba, N. (1993). Evaluation quantitative et qualitative des effluents et de polluants ; cas de la Côte d'Ivoire et de la ville d'Abidjan. Centre de Recherche Océanologiques, Abidjan, Côte d'Ivoire, 94 p.

15. Müller, G. (1969). Index of geoaccumulation in sediments of the Rhine River. Geojournal, Vol.2, pp. 109-118.

16. Soro, G., Soro, M.B., Soro, N., Ahoussi, K.E., Kouame, K.F., Zade, S.G.P. \& Soro, T. (2009). Métaux lourds (Cu, Cr, Mn et Zn) dans les sédiments de surface d'une lagune tropicale africaine : cas de la lagune Ebrié (Côte d’Ivoire). Int. J. Biol. Chem. Sci., Vol.3, Nº6, pp. 14081427.

17. Sutherland, R. A. (2000). Bed sediment associated trace metals in an urban stream, Oahu, Hawaii. Environmental Geology, Vol.39, pp. 611637.

18. Tastet, J.P. \& Guiral, D. (1994). Géologie et sédimentologie. in: Environnement et ressources aquatiques de Côte d'Ivoire, tome II. Les milieux lagunaires, Edition ORSTOM, pp. 35- 58.

19. Varlet F. (1978). Le régime de la lagune Ébrié (Côte d’Ivoire). Traits physiques essentiels. Travaux et documents ORSTOM. N83, 163p.

20. Wedepohl, K.H. (1995). The composition of continental crust. Goechimica and Cocmochimica Acta, Vol.59, Nº7, pp. 1217-1232. 